\title{
BMJ Open Effectiveness and safety of brain- computer interface technology in the treatment of poststroke motor disorders: a protocol for systematic review and meta-analysis
}

Xiaolin Zhang (D) , ${ }^{1}$ Di Cao, ${ }^{2}$ Junnan Liu, ${ }^{3}$ Qi Zhang, ${ }^{4}$ Mingjun Liu (D) ${ }^{4}$

To cite: Zhang X, Cao D, Liu J, et al. Effectiveness and safety of brain-computer interface technology in the treatment of poststroke motor disorders: a protocol for systematic review and

meta-analysis. BMJ Open 2021;11:e042383. doi:10.1136/ bmjopen-2020-042383

- Prepublication history for this paper is available online. To view these files, please visit the journal online (http://dx.doi. org/10.1136/bmjopen-2020042383).

Received 03 July 2020 Revised 08 December 2020 Accepted 14 December 2020

Check for updates

(C) Author(s) (or their employer(s)) 2021. Re-use permitted under CC BY-NC. No commercial re-use. See rights and permissions. Published by BMJ.

For numbered affiliations see end of article.

Correspondence to

Professor Mingjun Liu;

mingjunliu646590@163.com

\section{ABSTRACT}

Introduction About $85 \%$ of stroke survivors have upper extremity dysfunction, and more than $60 \%$ have continuing hand dysfunction and cannot live independently after treatment. Numerous recent publications have explored brain-computer interfaces technology as rehabilitation tools to help subacute and chronic stroke patients recover upper extremity movement. Our study aims to synthesise results from randomised controlled trials to assess the effectiveness and safety of brain-computer interface technology in the treatment of poststroke motor disorders ( PSMD ) .

Methods and analysis English and Chinese search strategies will be conducted in eight databases: the China National Knowledge Infrastructure, Chinese Scientific Journal Database, Wanfang Database, China Doctoral Dissertations Full-Text Database, China Master's Theses Full-Text Database, Cochrane Central Register of Controlled Trials, PubMed and Embase. In addition, manual retrieval of research papers, conference papers, ongoing experiments and internal reports, among others, will supplement electronic retrieval. The searches will select all eligible studies published on or before 8 June 2020. To enhance the effectiveness of the study, only randomised controlled trials related to brain-computer interface technology for poststroke motor disorders will be included. The Fugl-Meyer Motor Function score will be the primary outcome measure; the Modified Barthel Index, Modified Ashworth Score and the upper extremity freehand muscle strength assessment will be secondary outcomes. Side effects and adverse events will be included as safety evaluations. To ensure the quality of the systematic evaluation, study selection, data extraction and quality assessment will be independently performed by two authors, and a third author will handle any disagreement. Review Manager V.5.3.3 and STATA V.15.1 will be used to perform the data synthesis and subgroup analysis. Ethics and dissemination This systemic review will evaluate the efficacy and safety of brain-computer interface technology combined with routine rehabilitation treatment for treatment of poststroke motor disorders. Since all included data will be obtained from published articles,the review does not require ethical approval. The review will be published in a peer-reviewed journal. PROSPERO registration number CRD42020190868.
Strengths and limitations of this study

- Include randomised controlled trials to discover whether the source of motor recovery derives from conventional therapy, the motor imagery by itself, neurofeedback from brain-computer interface (BCI) or a combination of these.

- Language and publication date will not be restricted.

- Conduct the sensitivity analysis to test whether the conclusions are robust.

- Different types of $\mathrm{BCl}$ technology may lead to a large degree of heterogeneity.

- Subgroup analysis will be conducted to exclude differences related to the study location or length of treatment.

\section{INTRODUCTION}

Stroke or cerebrovascular accident is the second leading cause of death and disability in the world. ${ }^{1}$ In China, the incidence rate is the first, the number of new cases is over 2.5 million every year, the death toll is over 1.5 million and the cost of treatment is as high as 40 billion every year. ${ }^{2}$ About $85 \%$ of survivors have upper extremity dysfunction, ${ }^{3}$ and more than $60 \%$ still have hand dysfunction and cannot live independently after treatment. ${ }^{4}$ Such problems place a heavy burden on the family and society.

Although clinical diagnosis and treatment of stroke have improved in recent years, most surviving patients are left with motor, sensory, cognitive, language and mental disorders. Evidence-based medicine has shown that stroke rehabilitation is the most effective way to reduce disability, and is alsoa keylinkin the organisational management mode of stroke. ${ }^{6}$ Effective rehabilitation treatment can both restore the patients' residual function, and mobilise their potential, improving their ability to live independently and return to normallife. ${ }^{7}$ High-intensity, high-dose 
medicine and repeated related training tasks are key factors in poststroke rehabilitation treatment. ${ }^{5}$ In addition to traditional rehabilitation therapy, which relies on rehabilitation physiotherapists to train patients, auxiliary training with the help of a rehabilitation robot is also available. However, the training process can be boring and it is difficult to mobilise patients to participate in training, and the clinical evidencebased evidence indicates that the treatment effect is limited. ${ }^{8}$

With the continuous integration, promotion and development of rehabilitation medicine, biomedical engineering, computer science, artificial intelligence and other disciplines, brain-computer interface technology (BCIT) have successfully been used for motor recovery training in stroke patients. BCIT, a neuromodulation technique that includes virtual reality (VR), BCI, brain stimulation and neurofeedback, among other techniques, is a cutting-edge, popular and noninvasive new method of central nervous system intervention. It involves neuroplasticity, and has been studied and applied in clinical treatment. ${ }^{90}$ Some independent studies have shown that BCIT has better rehabilitation effects and is more interesting for patients than traditional rehabilitation because of its novelty. ${ }^{11}$ BCIT can be regarded as an auxiliary technology for people who are unable to communicate or are paralysed (eg, patients with poststroke limb dyskinesia, amyotrophic lateral sclerosis or spinal cord injury). It detects brain signals that convey intention and converts them into executable output through machines, making it 'a direct connection between living nerve tissue and artificial devices, establishing a communication channel between the computer and the brain'. ${ }^{12}$ Unlike related devices, BCIT involves two-way feedback between the user and the system to produce body changes, restoring some function for those who have lost limbs, suffered from massive paralysis or have severe neurological damage. Interface technology includes 'reading' the brain, which records brain activity and back to the brain in feedback manner, in order to manipulate the activity of a specific area and influence function. BCIT essentially involves translating human brain activity into external action by sending neural commands to external devices. ${ }^{12-17}$ Applying BCIT to rehabilitate upper limb and hand function in patients with stroke with hemiplegia is safer, less labour intense and allows patients to actively participate in rehabilitation training to promote central nervous system remodelling. ${ }^{18}$ This both facilitates effective recovery of hand function and improves the patients' ability to perform normal daily activities. ${ }^{19}$

The proposed date for completing the study is: 12 March 2021.

\section{METHODS AND ANALYSIS}

\section{Design and registration of the review}

The protocol is structured in accordance with the guidelines from the Preferred Reporting Items for Systematic Reviews and Meta-analyses Protocols (PRISMA-P). ${ }^{20}$

\section{Inclusion criteria for study selection}

Types of studies

Only randomised controlled trials (RCTs) that were published or registered before 8 June 2020 will be included. Quasi-RCTs, review articles, case reports and other studies that do not meet the requirements will be excluded.

\section{Types of patients}

Patients' age will be between 18 and 75 years. In line with China' s 2015 diagnostic criteria for classifying cerebrovascular diseases, the first stroke confirmed by CT or MRI with a course greater than 1 month and less than or equal to 6 months, with moderate to severe upper extremity and hand dysfunction meeting the criteria (Brunnstrom grade 2-4, the Fugl-Meyer Motor Function score (FMA) $<20$, Modified Ashworth Scale (MAS) less than level 3) will be included, regardless of gender and nationality. Patient exclusion criteria will include: presence of other diseases resulting in serious cognitive or speech disorders; patients who could not understand and complete the therapist's instructions (Mini-Mental State Examination <21 points); history of drug or alcohol dependence; serious liver or kidney disease; other diseases that may affect brain structure and function; and other mental disorders.

\section{Interventions types}

We will include studies where the intervention group received BCIT (including one or more of VR, BCI, brain stimulation and nerve feedback, among others), alone or in combination with routine rehabilitation treatment (manual therapy, exercise therapy and electronic biofeedback, among others), while the control group received only conventional rehabilitation treatment.

\section{Outcome measures}

Primary outcome

The primary outcome measure will be the FMA score.

\section{Secondary outcomes}

Secondary outcomes will include the Modified Barthel Index, MAS and the upper extremity freehand muscle strength assessment.

In the subgroup analyses, times to improvement of motor function and the type of therapeutic intervention combined with BCIT will be analysed.

\section{Article exclusion criteria}

Studies with the following situations will be excluded: participants were diagnosed with secondary stroke; duplicated data or data that cannot be extracted; observational studies; retrospective studies; non-randomised trials; quasiexperimental studies and animal studies. Additionally, studies with insufficient data or lacking effective sort will also not be included.

\section{Search methods for the identifying of studies}

English and Chinese search strategies will be conducted on eight databases: the China National Knowledge Infrastructure, Chinese Scientific Journal Database, Wanfang Database, China Doctoral Dissertations Full-Text Database and China Master's Theses Full-Text Database, Cochrane Central Register of Controlled Trials, PubMed 


\begin{tabular}{cl}
\hline Table 1 & Search strategy for PubMed \\
\hline No & Search terms \\
\hline 1 & brain computer interface.ti,mesh. \\
\hline 2 & direct neural interface.ti,ab. \\
\hline 3 & neurofeedback.ti,ab. \\
\hline 4 & brain-machine interface.ti,ab. \\
\hline 5 & or $1-4$ \\
\hline 7 & post-stroke. ti,ab. \\
\hline 8 & after stroke. ti,ab. \\
\hline 9 & or $6-7$ \\
\hline 10 & motor disorders. ti,mesh. \\
\hline 11 & dyskinesias. ti,ab. \\
\hline 12 & or $9-10$ \\
\hline 13 & randomised controlled trial.pt. \\
\hline 14 & Controlled clinical trial.pt. \\
\hline 15 & randomised.ab. \\
\hline 16 & Randomly.ab. \\
\hline 17 & trial.ab. \\
\hline 18 & or $12-16$ \\
\hline
\end{tabular}

and Embase. In addition, we will conduct manual retrieval of papers, conference papers, ongoing experiments and internal reports, among others, to supplement electronic retrieval. We will select all eligible studies published on or before 8 June 2020.

\section{Search strategy}

The search strategy will be based on the Cochrane handbook guidelines (V.5.1.0) including keywords such as 'poststroke', 'motor disorders', 'brain computer interface' or 'neurofeedback' and 'RCT'. Subsequent searches will use Medical Subject Headings ( MeSH ) headings, including 'poststroke', 'motor disorders', and 'brain computer interface', in addition to keywords from the initial retrieval. Additional article searches will review the reference lists of relevant research articles. As an example, the search strategy for PubMed is summarised in table 1 .

\section{Data extraction}

Study selection

Records from databases and other resources will be uploaded to a database created in EndNote, V.9.7. All extracted abstracts will be independently screened by the review authors (XZ and JL). We will obtain the full text of all potentially suitable articles to further assess eligibility based on the inclusion/exclusion criteria. Studies that do not meet the inclusion criteria will be excluded and the reason for exclusion will be recorded. Any disagreement will be resolved by consensus or discussion with a third author (ML). The final selection procedure will follow the PRISMA guidelines, ${ }^{21}$ and is presented in figure 1.

\section{Data extraction and management}

Two reviewers (XZ and QZ) will independently apply the inclusion and exclusion criteria to assess each retrieved study's eligibility. The following data will then be extracted from the selected studies for inclusion using a data collection form and recorded in an Excel file: first author and publication year, study design, sample, intervention, types of measures, risk of bias assessment and findings.

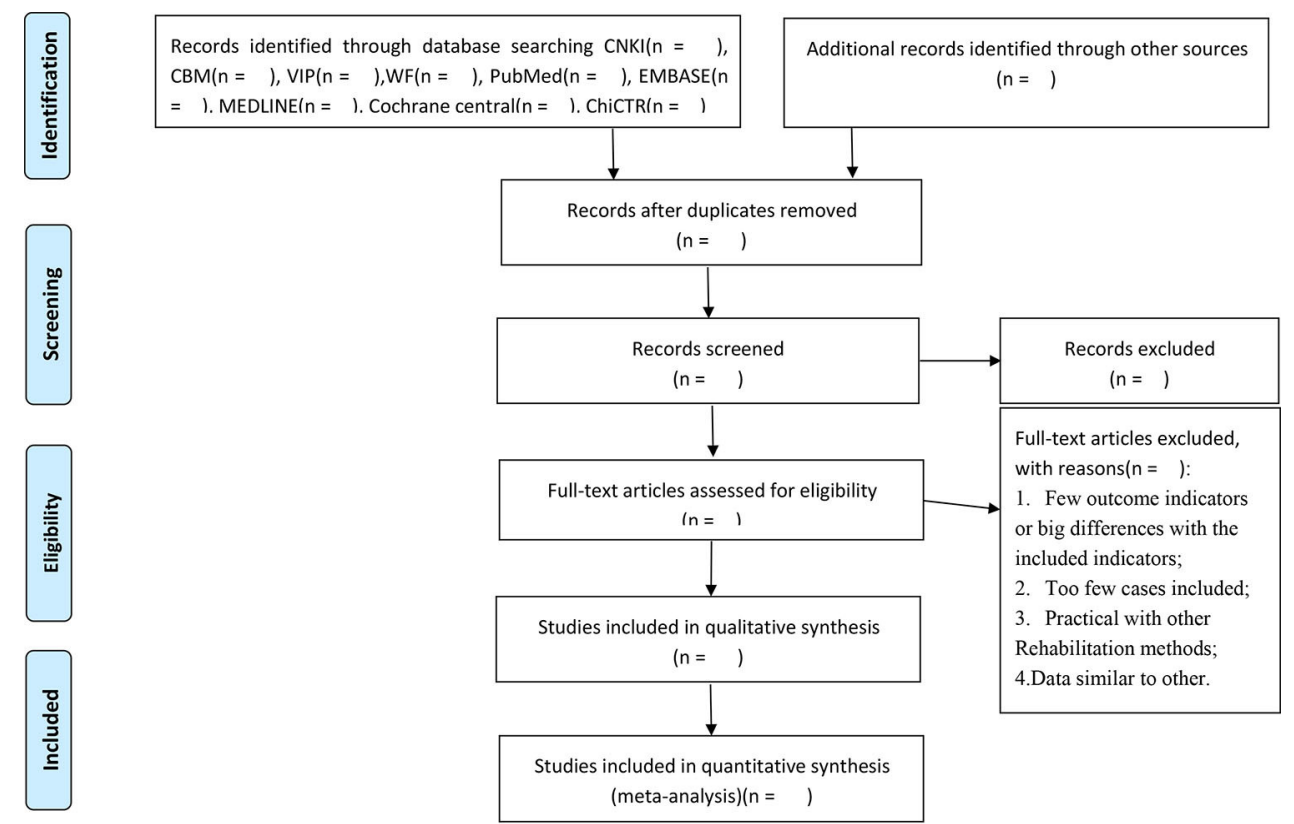

Figure 1 PRISMA flow diagram of study and exclusion. PRISMA, Preferred Reporting Items for Systematic Reviews and Metaanalyses. 
The results will be cross-checked by the two reviewers, and disagreements will be resolved by consensus, with any ongoing differences in opinion arbitrated by a third reviewer (DC). We may also contact the original authors to provide additional relevant information, if necessary.

The data extraction form will include the following items:

1. General information: title, authors, publication year and study area, average patient age, average disease course and treatment time.

2. Trial characteristics: design, follow-up duration, randomisation method, allocation concealment, incomplete outcome data, blinding (patients, people administering treatment, outcome assessors).

3. Intervention: primary intervention (BCIT type, routine rehabilitation treatment, application time, session duration); comparison interventions (routine rehabilitation treatment, application time, session duration).

4. Patients: total number and number in each group, baseline characteristics, diagnostic criteria, withdrawals and losses to follow-up (reasons, description).

5. Outcomes: primary outcomes, adverse drug reactions, adverse time, follow-up length, quality of outcomes reporting.

\section{Risk of bias in assessment}

Two reviewers (XZ and DC) will independently apply the bias tool from the Cochrane Handbook for Systematic Reviews of Interventions ${ }^{22}$ to evaluate the risk of bias in each selected study. Six dimensions will be assessed: random sequence generation; allocation concealment; blinding method for patients, researchers and outcome evaluators; incomplete results data; selective reporting and other issues. The studies will be categorised into three quality levels: low risk of bias, high risk of bias and unclear risk of bias. ${ }^{20}$ Any discrepancies will be resolved through discussions with the third author. When a consensus cannot be reached by discussion, the third reviewer (ML) will decide.

\section{Treatment effect measures}

Methods will vary by data type. For dichotomous variables, total effective rate and adverse events, we will analyse the rate ratio; for continuous variables, we will analyse mean differences. The $95 \%$ CI will be presented for both dichotomous and continuous outcomes.

\section{Missing data management}

We will contact the original author to obtain the missing or incomplete data and will wait 1 month after an email is sent to receive a reply. If we are unable to obtain the missing data, the incomplete data will be excluded from the analysis

\section{Heterogeneity assessment}

Statistical heterogeneity will be assessed with the $\mathrm{I}^{2}$ statistic. ${ }^{23}$ An I ${ }^{2}$ statistic of less than $50 \%$ indicates a low level of statistical heterogeneity; $50 \%$ or more will be considered substantial statistical heterogeneity. If substantial heterogeneity is identified, we will report it and explore possible causes using sensitivity analysis and subgroup analysis.

\section{Reporting biases assessment}

We will construct funnel plots to evaluate reporting bias if the included studies include more than ten trials. Otherwise, STATA V.15.1 software will be used to perform the Egger's test.

\section{Subgroup analysis}

We plan to carry out the following subgroup analyses, if possible: study area differences, differences in routine rehabilitation methods, average course of disease differences and length of treatment differences. We will use the formal test for subgroup interactions in Review Manager V.5.3.

\section{Sensitivity analysis}

When possible, we will perform sensitivity analysis to explore the effects of the trial's bias risk on primary outcomes. These analyses will exclude lower quality trials and repeat the meta-analyses to assess quality and robustness when significant statistical heterogeneity arises, according to sample size and insufficient data.

\section{Grading the quality of evidence}

The online version of the Grading of Recommendations Assessment, Development, and Evaluation methodology (GRADE; https://www.gradeworkinggroup.org/) ${ }^{24}$ will be used to assess the quality of the evidence and risk of bias, categorised into four levels: high, moderate, low or very low.

\section{Ethics and dissemination}

This systemic review will evaluate the efficacy and safety of BCIT combined with routine rehabilitation for treatmen of PSMD. Since all included data will be obtained from published articles,it does not require ethical approval, and will be published in a peer-reviewed journal. Due to the lack of relevant systematic reviews in this field, this study will combine relevant RCTs to better explore evidence on BCIT combined with routine rehabilitation for treatment of PSMD and guide clinical practice and BCIT researches.

\section{Patient and public involvement}

This article is based on previously conducted studies and does not involve any patient and public involvement or new studies of human subjects performed by any of the authors.

\section{DISCUSSION}

With the ageing of the world population and the influence of living habits and environmental changes, stroke has become a major global health issue..$^{25}$ Motor dysfunction of the upper limbs and hands following stroke is especially important in clinical settings because of its 
long treatment cycle and poor prognosis. ${ }^{26}$ Currently, most conventional upper limb and hand function rehabilitation techniques following stroke focus on peripheral treatment, such as facilitation techniques, functional electrical stimulation, but these methods often result in low treatment effectiveness, so they are insufficient. ${ }^{27} 28$

Recent developments in rehabilitation medicine and artificial intelligence technology have focused on more direct brain-based interventions. ${ }^{29}$ BCIT employs control signals generated by Electroencephalogram(EEG) activities, allowing patients to interact with the surrounding environment without the influence of peripheral nerves and muscles. This can effectively overcome the limitations of traditional rehabilitation methods, increase patients' interest in treatment and benefit upper limb and hand function rehabilitation. BCIT applied in the rehabilitation of motor disorders after stroke is safer, requires less time and work for clinicians, and allows patients to actively participate in rehabilitation training that promotes remodelling of the central nervous system. ${ }^{30}$ BCIT is a promising treatment for recovering hand function following stroke, which will facilitate patients' ability to perform activities of daily life. ${ }^{31}$

However, the specific BCIT mechanisms that facilitate rehabilitation of poststroke limb disorders needs further research, because it is not clear whether the source of motor recovery derives from conventional therapy, motor imagery by itself, neurofeedback from BCI, or a combination of these mechanisms. This systematic review and meta-analysis will provide patients, clinicians and healthcare policy-makers with a deeper understanding of BCIT' s efficacy and safety in the treatment of PSMD. The PRISMA-P checklist of this protocol is presented in PRISMA-P checklis.

\author{
Author affiliations \\ ${ }^{1}$ College of acupuncture and massage, Changchun University of Chinese Medicine, \\ Changchun, China \\ ${ }^{2}$ Department of rehabilitation, the Second Affiliated Hospital of Changchun \\ University of Chinese Medicine ( Changchun Hospital of Chinese Medicine ), \\ Changchun, China \\ ${ }^{3}$ Department of lung diseases, the Third Affiliated Hospital of Changchun University \\ of Chinese Medicine, Changchun, China \\ ${ }^{4}$ Changchun University of Chinese Medicine, Changchun, China
}

Contributors $X Z$ conceived the study and provided general guidance to the drafting of the protocol. XZ and DC drafted the protocol. QZ designed the search strategy. XZ, DC, JL, QZ and ML drafted the manuscript. ML and XZ reviewed and revised the manuscript. All authors have read and approved the final version of the manuscript.

Funding This work was supported by the School construction project of State Administration of traditional Chinese medicine (grant number LPGZS22014-11) and The Jilin Local Standard Construction Project (grant number: DBXM097-2020).

Competing interests None declared.

Patient and public involvement Patients and/or the public were not involved in the design, or conduct, or reporting, or dissemination plans of this research.

Patient consent for publication Not required.

Provenance and peer review Not commissioned; externally peer reviewed.

Open access This is an open access article distributed in accordance with the Creative Commons Attribution Non Commercial (CC BY-NC 4.0) license, which permits others to distribute, remix, adapt, build upon this work non-commercially, and license their derivative works on different terms, provided the original work is properly cited, appropriate credit is given, any changes made indicated, and the use is non-commercial. See: http://creativecommons.org/licenses/by-nc/4.0/.

\section{ORCID iDs}

Xiaolin Zhang http://orcid.org/0000-0002-9875-1931

Mingjun Liu http://orcid.org/0000-0001-9643-0184

\section{REFERENCES}

1 Wu S, Wu B, Liu M, et al. Stroke in China: advances and challenges in epidemiology, prevention, and management. Lancet Neurol 2019;18:394-405.

2 Wang Y-J, Li Z-X, Gu H-Q, et al. China stroke statistics 2019: a report from the National center for healthcare quality management in neurological diseases, China national clinical research center for neurological diseases, the Chinese stroke association, National center for chronic and non-communicable disease control and prevention, Chinese center for disease control and prevention and Institute for global neuroscience and stroke collaborations. Stroke Vasc Neurol 2020;5:211-39.

3 Broeks JG, Lankhorst GJ, Rumping K, et al. The long-term outcome of arm function after stroke: results of a follow-up study. Disabil Rehabil 1999;21:357-64.

4 Plantin J, Laurencikas E, Lindberg P, et al. Biomarkers for prediction of hand function after stroke: the ProHand study protocol. $J$ Neurol Sci 2013;333:e555-6.

5 Tabernig CB, Lopez CA, Carrere LC, et al. Neurorehabilitation therapy of patients with severe stroke based on functional electrical stimulation commanded by a brain computer interface. J Rehabil Assist Technol Eng 2018;5:205566831878928.

6 Winstein CJ, Stein J, Arena R, et al. Guidelines for adult stroke rehabilitation and recovery: a guideline for healthcare professionals from the American heart Association/American stroke association. Stroke 2016;47:e98-169.

7 Zhang XY, JH X, YJ S. Investigation and research on continuous nursing needs of subjects with primary stroke. Chinese Nurs Educat 2012;9:294-6.

8 Neurology branch of Chinese Medical Association, neurorehabilitation group of Neurology branch of Chinese Medical Association, Cerebrovascular disease group of Neurology branch of Chinese Medical Association. Guidelines for early rehabilitation of stroke in China. Chinese J Neurol 2017;50:405-12.

9 Rosenfeld JV, Wong YT, Jv R. Neurobionics and the brain-computer interface: current applications and future horizons. Med J Aust 2017;206:363-8.

10 Salisbury DB, Parsons TD, Monden KR, et al. Brain-computer interface for individuals after spinal cord injury. Rehabil Psychol 2016;61:435-41.

11 Kasashima-Shindo Y, Fujiwara T, Ushiba J, et al. Brain-computer interface training combined with transcranial direct current stimulation in patients with chronic severe hemiparesis: proof of concept study. J Rehabil Med 2015;47:318-24.

12 Grigoryan KA, Nikulin V, Anwander A, et al. P29 neural correlates of post-stroke rehabilitation based on brain-computer interfaces: preliminary outlook. Clinical Neurophysiology 2020;131:e192-3.

13 Yan Z, Zhang LX. Application of brain computer interface in rehabilitation therapy. Chinese J Rehab Med 2020;35:228-32.

14 Liang SJ, Zhu YL, Wang WN. The application of brain computer interface technology in the rehabilitation of upper limb dysfunction of stroke patient. Chinese J Rehab Med 2020;35:185-8.

15 Wu Q, Ren SY, Le Z. Study on the effect of brain computer interface comprehensive rehabilitation training on subacute stroke with resting functional MRI. Chinese Rehab Theory Pract 2020;26:77-84.

16 Tsuchimoto S, Shindo K, Hotta F, et al. Sensorimotor connectivity after motor exercise with neurofeedback in post-stroke patients with hemiplegia. Neuroscience 2019;416:109-25.

17 Carvalho R, Dias N, Cerqueira JJ. Brain-machine interface of upper limb recovery in stroke patients rehabilitation: a systematic review. Physiother Res Int 2019;24:e1764.

18 Birbaumer N. Breaking the silence: brain-computer interfaces (BCl) for communication and motor control. Psychophysiology 2006;43:517-32.

19 Fang WN, Liu H. Application of brain computer interface technology in the rehabilitation of lower extremity motor function of stroke hemiplegia patients. Shandong Med 2018;58:66-8.

20 Moher D, Shamseer L, Clarke M, et al. Preferred reporting items for systematic review and meta-analysis protocols (PRISMA-P) 2015 statement. Syst Rev 2015;4:1-9. 
21 Liberati A, Altman DG, Tetzlaff J, et al. The PRISMA statement for reporting systematic reviews and meta-analyses of studies that evaluate health care interventions: explanation and elaboration. PLoS Med 2009;6:e1000100.

22 Higgins J, Green S. Cochrane handbook for systematic reviews of interventions version 5.1.0, 2011. Available: http://handbook-5-1. cochrane.org/

23 Higgins JPT, Thompson SG, Deeks JJ, et al. Measuring inconsistency in meta-analyses. BMJ 2003;327:557-60.

24 HY M, Yang ZF, Zheng JP. Experimental study on the prevention and treatment of FM1 infected mice by Lianhuaqingwen capsule [J]. Chinese Herbal Med 2008;31:1230-3.

25 Sun HX, Wang WZ. Epidemiological sampling report of cerebrovascular diseases among 600000 people in China. China $J$ Modern Neurol Dis 2018;18:83-8.

26 Alberts MJ, Latchaw RE, Jagoda A, et al. Revised and updated recommendations for the establishment of primary stroke centers: a summary statement from the brain attack coalition. Stroke 2011;42:2651-65.

27 Thieme H, Mehrholz J, Pohl M, et al. Mirror therapy for improving motor function after stroke. Stroke 2013;44:e1-2.

28 Cervera MA, Soekadar SR, Ushiba J, et al. Brain-computer interfaces for post-stroke motor rehabilitation: a meta-analysis. Ann Clin Trans/ Neurol 2018;5:651-63.

29 Zhu YL. Intelligent development of rehabilitation technology. Shanghai Pharmaceut 2017;38:9-12.

30 Birbaumer N. Breaking the silence: brain-computer interfaces $(\mathrm{BCl})$ for communication and motor control. Psychophysiology 2006;43:517-32

31 Jochumsen M, Knoche $\mathrm{H}$, Kidmose P, et al. Evaluation of EEG Headset mounting for brain-computer Interface-Based stroke rehabilitation by patients, therapists, and relatives. Front Hum Neurosci 2020;14:13 